ANALYSIS \& PDE Volume $8 \quad$ No. $2 \quad 2015$ (3)

GIOVANNi CATINo, CARLO MANTEGAZZA AND LORENZO MAZZIERI LOCALLY CONFORMALLY FLAT ANCIENT RICCI FLOWS

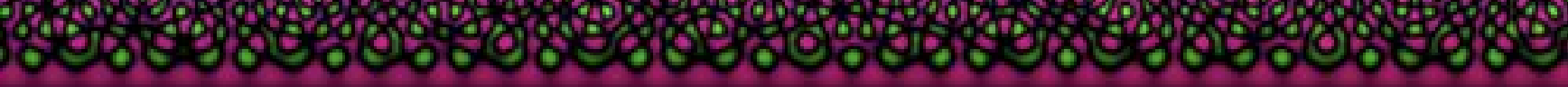




\title{
LOCALLY CONFORMALLY FLAT ANCIENT RICCI FLOWS
}

\author{
Giovanni Catino, CARlo MantegazZa And Lorenzo MaZzieri
}

We show that any locally conformally flat ancient solution to the Ricci flow must be rotationally symmetric. As a by-product, we prove that any locally conformally flat Ricci soliton is a gradient soliton in the shrinking and steady cases as well as in the expanding case, provided the soliton has nonnegative curvature.

\section{Introduction}

In this paper, we study ancient solutions to the Ricci flow. We recall that a time-dependent metric $g(t)$ on a Riemannian manifold $M$ is a solution to the Ricci flow if it evolves by the equation

$$
\frac{\partial}{\partial t} g(t)=-2 \operatorname{Ric}_{g(t)} \text {. }
$$

A solution is called ancient if it is defined for every negative time. Ancient solutions typically arise as the limit of a sequence of suitable blow-ups as the time approaches a singular time for the Ricci flow. In dimension two there exists a compact, rotationally symmetric, ancient solution due to King [1990], Rosenau [1995] and Fateev, Onofri and Zamolodchikov [Fateev et al. 1993]. In dimension three, Perelman [2003] constructed a compact, rotationally symmetric, ancient solution on the three-sphere. In the nonrotationally symmetric case, the first construction is due to Fateev [1996] in dimension three. Motivated by this construction, Bakas, Kong and Ni [Bakas et al. 2012] produced high-dimensional, compact, ancient solutions to the Ricci flow which are not rotationally symmetric.

In dimension two, Daskalopoulos, Hamilton and Sesum [Daskalopoulos et al. 2012] have obtained a complete classification of all compact ancient solutions to the Ricci flow. Ni [2010] showed that any compact ancient solution to the Ricci flow which is of type I is $k$-noncollapsed and has positive curvature operator has constant sectional curvature. Brendle, Huisken and Sinestrari [Brendle et al. 2011] proved that any compact ancient solution which satisfies a suitable pinching condition must have constant sectional curvature.

In this article, we show that any complete ancient solution to the Ricci flow in dimension $n \geq 4$ which is locally conformally flat along the flow must be rotationally symmetric.

Theorem 1.1. Let $\left(M^{n}, g(t)\right), n \geq 4$, be a complete ancient solution to the Ricci flow which is locally conformally flat at every time. Then $\left(M^{n}, g(t)\right)$ is rotationally symmetric.

MSC2010: 53C24, 53C25, 53C44.

Keywords: Ricci solitons, Ricci flow. 
The nonrotationally symmetric examples of Bakas, Kong and Ni show that the locally conformally flatness assumption cannot be removed. The proof of Theorem 1.1 relies on previous work of Catino and Mantegazza [2011] about the behavior of the Weyl tensor under the Ricci flow, combined with a more recent result [Catino et al. 2014] concerning the classification of Riemannian manifolds admitting a Codazzi tensor with exactly two distinct eigenvalues.

As a consequence of Theorem 1.1, we classify locally conformally flat Ricci solitons. We recall that a complete Riemannian manifold $\left(M^{n}, g\right)$ is a Ricci soliton if there exists a vector field $X$ on $M^{n}$ such that

$$
\operatorname{Ric}+\frac{1}{2} \mathscr{L}_{X} g=\lambda g
$$

for some constant $\lambda$. The Ricci soliton is called shrinking if $\lambda>0$, steady if $\lambda=0$, and expanding if $\lambda<0$. If $X=\nabla f$ for some smooth function $f$, then the soliton is called a gradient Ricci soliton. It follows from the work of Perelman [2002] (see [Eminenti et al. 2008], for instance, for a direct proof) that any compact Ricci soliton is actually a gradient soliton. Moreover, Naber [2010] has shown that any shrinking Ricci soliton with bounded curvature has a gradient soliton structure. On the other hand, steady and expanding Ricci solitons which do not support a gradient structure were found by [Lauret 2001; Baird and Danielo 2007; Lott 2007; Baird 2009].

In this article we prove the following result:

Theorem 1.2. Let $\left(M^{n}, g, X\right), n \geq 4$, be a complete, locally conformally flat, shrinking or steady Ricci soliton. Then it is a gradient Ricci soliton. The conclusion still holds in the expanding case provided the soliton has nonnegative curvature operator.

In particular, from the classification results of locally conformally flat gradient Ricci solitons in the shrinking case [Cao et al. 2011; Eminenti et al. 2008; Ni and Wallach 2008; Petersen and Wylie 2010; Zhang 2009a], in the steady case [Cao and Chen 2012; Catino and Mantegazza 2011], as well as in the expanding case [Catino and Mantegazza 2011], we obtain the following corollaries:

Corollary 1.3. Let $\left(M^{n}, g, X\right), n \geq 4$, be a complete, locally conformally flat, shrinking Ricci soliton. Then it is isometric to a quotient of $\mathbb{S}^{n}, \mathbb{R} \times \mathbb{S}^{n-1}$ or $\mathbb{R}^{n}$.

Corollary 1.4. Let $\left(M^{n}, g, X\right), n \geq 4$, be a complete, locally conformally flat, steady Ricci soliton. Then it is isometric to a quotient of $\mathbb{R}^{n}$ or the Bryant soliton.

Corollary 1.5. Let $\left(M^{n}, g, X\right), n \geq 4$, be a complete, locally conformally flat, expanding Ricci soliton with nonnegative curvature operator. Then it is a rotationally symmetric gradient expanding Ricci soliton.

We note that rotationally symmetric gradient expanding Ricci solitons were constructed in [Bryant 2005; Cao 1997; Feldman et al. 2003].

\section{Notations and preliminaries}

The Riemann curvature operator of a Riemannian manifold $\left(M^{n}, g\right)$ is defined as in [Gallot et al. 1990] by

$$
\operatorname{Riem}(X, Y) Z=\nabla_{Y} \nabla_{X} Z-\nabla_{X} \nabla_{Y} Z+\nabla_{[X, Y]} Z
$$


In a local coordinate system the components of the $(3,1)$-Riemann curvature tensor are given by $\mathrm{R}_{i j k}^{l} \partial / \partial x^{l}=\operatorname{Riem}\left(\partial / \partial x^{i}, \partial / \partial x^{j}\right) \partial / \partial x^{k}$ and we denote by $\mathrm{R}_{i j k l}=g_{l m} \mathrm{R}_{i j k}^{m}$ its $(4,0)$-version. With this choice, we have that the round sphere $\mathbb{S}^{n}$ has positive curvature, meaning that

$$
\operatorname{Riem}(v, w, v, w)=\mathrm{R}_{i j k l} v^{i} w^{j} v^{k} w^{l}>0
$$

for every couple $u$ and $v$ of nonparallel vector fields. The Ricci tensor is obtained by the contraction $\mathrm{R}_{i k}=g^{j l} \mathrm{R}_{i j k l}$, and $\mathrm{R}=g^{i k} \mathrm{R}_{i k}$ will denote the scalar curvature. The so-called Weyl tensor is then defined by the following decomposition formula (see [Gallot et al. 1990, Chapter 3, Section K]) in dimension $n \geq 3$ :

$$
\mathrm{W}_{i j k l}=\mathrm{R}_{i j k l}+\frac{\mathrm{R}}{(n-1)(n-2)}\left(g_{i k} g_{j l}-g_{i l} g_{j k}\right)-\frac{1}{n-2}\left(\mathrm{R}_{i k} g_{j l}-\mathrm{R}_{i l} g_{j k}+\mathrm{R}_{j l} g_{i k}-\mathrm{R}_{j k} g_{i l}\right) .
$$

The Weyl tensor shares the symmetries of the curvature tensor. Moreover, as can be easily seen by the formula above, all of its contractions with the metric are zero.

In dimension three, $\mathrm{W}$ is identically zero on every Riemannian manifold, whereas, when $n \geq 4$, the vanishing of the Weyl tensor is a relevant condition, since it is equivalent to the locally conformally flatness of $\left(M^{n}, g\right)$. We recall that this latter condition means that around every point $p \in M^{n}$ there exists a smooth function $f$ defined in a open neighborhood $U_{p}$ of $p$ such that the conformal deformation $\tilde{g}$ of the original metric $g$ defined by $\tilde{g}_{i j}=e^{f} g_{i j}$ is flat. In particular, the Riemann tensor associated to $\tilde{g}$ is zero in $U_{p}$.

We also recall that, in dimension $n=3$, locally conformally flatness is equivalent to the vanishing of the Cotton tensor

$$
\mathrm{C}_{i j k}=\nabla_{k} \mathrm{R}_{i j}-\nabla_{j} \mathrm{R}_{i k}-\frac{1}{2(n-1)}\left(\nabla_{k} \mathrm{R} g_{i j}-\nabla_{j} \mathrm{R} g_{i k}\right) .
$$

By direct computation, we can see that the tensor $\mathrm{C}_{i j k}$ satisfies the symmetries

$$
\mathrm{C}_{i j k}=-\mathrm{C}_{i k j}, \quad \mathrm{C}_{i j k}+\mathrm{C}_{j k i}+\mathrm{C}_{k i j}=0
$$

moreover, it is trace-free in any two indices:

$$
g^{i j} \mathrm{C}_{i j k}=g^{i k} \mathrm{C}_{i j k}=g^{j k} \mathrm{C}_{i j k}=0
$$

by its skew-symmetry and the Schur lemma. We note that, for $n \geq 4$,

$$
\nabla^{l} \mathrm{~W}_{i j k l}=-\frac{n-3}{n-2} \mathrm{C}_{i j k}
$$

and we refer the reader to [Besse 1988] for the detailed computation. It follows from this formula that, in every dimension $n \geq 3$, the vanishing of the Cotton tensor is a necessary condition for a Riemannian manifold $\left(M^{n}, g\right)$ to be locally conformally flat. We also note that the vanishing of the Cotton tensor can be rephrased in terms of the so-called Schouten tensor

$$
\mathrm{S}_{i j}=\mathrm{R}_{i j}-\frac{1}{2(n-1)} \mathrm{R} g_{i j}
$$


by saying that $\mathrm{S}$ must satisfy the Codazzi equation

$$
\left(\nabla_{X} \mathrm{~S}\right) Y=\left(\nabla_{Y} \mathrm{~S}\right) X, \quad X, Y \in T M
$$

Any symmetric two-tensor satisfying this condition is called a Codazzi tensor (see [Besse 1988, Chapter 16] for a general overview on Codazzi tensors). Hence, if $\left(M^{n}, g\right), n \geq 3$, is a locally conformally flat manifold, then the Schouten tensor is a Codazzi tensor.

\section{Proof of Theorem 1.1}

Let $\left(M^{n}, g(t)\right), n \geq 4$, be a complete ancient solution to the Ricci flow. We assume that, along the flow, the Weyl tensor remains identically zero. As was observed in [Catino and Mantegazza 2011], this condition implies a strong rigidity on the eigenvalues of the Ricci tensor. More precisely, one has the following result:

Lemma 3.1 [Catino and Mantegazza 2011, Corollary 1.2]. Let $\left(M^{n}, g\right), n \geq 4$, be a solution to the Ricci flow such that the Weyl tensor remains identically zero at every time. Then, at every point, either the Ricci tensor is proportional to the metric or it has an eigenvalue of multiplicity $n-1$ and another of multiplicity 1.

By the results in [Chen 2009; Zhang 2009a], which generalize the well-known Hamilton-Ivey curvature estimate, we know that every complete ancient solution $g(t)$ to the Ricci flow whose Weyl tensor is identically zero for all times is forced to have nonnegative curvature operator for every time $t$. Moreover, by Hamilton's strong maximum principle for systems in [Hamilton 1986], we have that either the metric has strictly positive curvature operator or it splits a line. By Theorem 1.167 in [Besse 1988], a Riemannian product $\left(\mathbb{R} \times N^{n-1}, d s \times h\right)$ is locally conformally flat if and only if the manifold $\left(N^{n-1}, h\right)$ has constant curvature; hence, one of the following possibilities holds: $\left(M^{n}, g(t)\right)$ is flat, or it is a quotient of a rescaling of $\mathbb{R} \times \mathbb{S}^{n-1}$, or it has positive curvature operator. Since the first two cases satisfy the conclusion of the theorem, from now on we assume that $\left(M^{n}, g(t)\right)$ is a complete, locally conformally flat, ancient solution to the Ricci flow with positive curvature operator.

As we have seen in the previous section, the relation (2-4) implies that the Cotton tensor is identically zero, hence the Schouten tensor

$$
\mathrm{S}_{i j}=\mathrm{R}_{i j}-\frac{1}{2(n-1)} \mathrm{R} g_{i j}
$$

is a Codazzi tensor. Moreover, from Lemma 3.1, we know that, at every point, either the metric is Einstein or the Ricci tensor (and so the Schouten tensor) has two distinct eigenvalues of multiplicity 1 and $n-1$, respectively. Now, it was proved by Bando [1987] that solutions to the Ricci flow are real analytic. To be precise, Bando showed that any Ricci flow solutions is real analytic if $M^{n}$ is compact or if it is complete with uniformly bounded curvature. This result was recently improved by Kotschwar [2013], who showed a local version of Bando's result. It follows that, if the metric is Einstein in some open subset of $M^{n}$, then it is Einstein everywhere and, by conformally flatness and positivity of the curvature, the manifold $\left(M^{n}, g\right)$ must be isometric to a quotient of $\mathbb{S}^{n}$. Thus, either $\left(M^{n}, g(t)\right)$ has constant positive sectional curvature 
or the Schouten tensor has an eigenvalue of multiplicity 1 and a different one of multiplicity $n-1$ at every point of some open dense subset $U$ of $M^{n}$. In the latter case, we apply to such an open set $U$ the following classification result of Riemannian manifolds admitting a Codazzi tensor with two distinct eigenvalues:

Lemma 3.2 [Catino et al. 2014, Theorem 2.1]. Let $\mathrm{T}$ be a Codazzi tensor on the Riemannian manifold $(U, g)$ with $n \geq 3$. Suppose that, at every point of $U$, the tensor $\mathrm{T}$ has exactly two distinct eigenvalues $\rho$ and $\sigma$, of multiplicity 1 and $n-1$, respectively. Finally, we let $W=\{p \in U \mid d \sigma(p) \neq 0\}$. Then we have that:

(1) The closed set $\bar{W}=W \cup \partial W$ with the metric $\left.g\right|_{\bar{W}}$ is locally isometric to the warped product of some $(n-1)$-dimensional Riemannian manifold on an interval of $\mathbb{R}$ and $\sigma$ is constant along the "leaves" of the warped product.

(2) The boundary of $W$, if present, is given by the disjoint union of connected, totally geodesic hypersurfaces where $\sigma$ is constant.

(3) Each connected component of the complement of $\bar{W}$ in $U$, if present, has $\sigma$ constant and it is foliated by totally geodesic hypersurfaces.

We are going to show that, under our assumptions, case (3) cannot occur and $W=U$. In fact, if a connected component of the complement of $\bar{W}$ in $U$ is present, the proof of this lemma (see also [Besse 1988, Proposition 16.11]) shows that the totally geodesic foliation of $(U, g(t))$ is integrable. Then, since the manifold $(U, g(t))$ has nonnegative sectional curvature, it follows from [Abe 1973, Corollary 2] that such a component must split a flat factor. This is clearly in contradiction with the positivity of the curvature, hence it must be that $U=W$ in this lemma and $(U, g(t))$ is locally a warped product of some $(n-1)$-dimensional manifold on an interval of $\mathbb{R}$. Since $(U, g(t))$ is locally conformally flat with positive curvature operator, we have that the $(n-1)$-dimensional fibers of the warped product are isometric to $\mathbb{S}^{n-1}$ and the metric is rotationally symmetric. By the density of $U$ in $M^{n}$, this conclusion clearly holds for the whole $\left(M^{n}, g\right)$. This concludes the proof of Theorem 1.1.

Remark 3.3. We would like to note that the same argument shows that the conclusion of Theorem 1.1 still holds if one consider a Ricci flow solution $\left(M^{n}, g(t)\right), n \geq 4$, defined on some time interval $I \subseteq \mathbb{R}$, which is locally conformally flat with nonnegative curvature operator for every $t \in I$.

\section{Proof of Theorem 1.2}

Now we turn our attention to the classification of locally conformally flat Ricci solitons. Let $\left(M^{n}, g, X\right)$ be a complete, locally conformally flat shrinking or steady Ricci soliton. In particular, it generates a self-similar ancient solution $g(t)$ to Ricci flow (see [Zhang 2009b]) which is locally conformally flat at every time $t$. Hence, Theorem 1.1 implies that the manifold is rotationally symmetric with nonnegative curvature operator. As we observed in Remark 3.3, the conclusion still holds if we consider an expanding Ricci soliton with nonnegative curvature operator. To prove Theorem 1.2 we then apply the following result: 
Lemma 4.1 [Catino and Mantegazza 2011, Proposition 2.6]. Let $\left(M^{n}, g, X\right)$ be a complete, locally warped, locally conformally flat Ricci soliton with nonnegative Ricci tensor; then it is a gradient Ricci soliton with a potential function $f: M^{n} \rightarrow \mathbb{R}$ (hence, $X=\nabla f$ ) depending only on the $r$ variable of the warping interval.

\section{Acknowledgments}

This work was partially supported by the Italian projects FIRB-IDEAS "Analysis and beyond" and GNAMPA projects "Flussi geometrici e soluzioni autosimilari" and "Equazioni di evoluzione geometriche e strutture di tipo Einstein". Mantegazza and Mazzieri were partially supported by the SNS project "Geometric flows and related topics". Mazzieri was also partially supported by the Italian project FIRB 2012 "Geometria Differenziale e Teoria Geometrica delle Funzioni".

\section{References}

[Abe 1973] K. Abe, "Applications of a Riccati type differential equation to Riemannian manifolds with totally geodesic distributions", Tôhoku Math. J. (2) 25 (1973), 425-444. MR 50 \#3163 Zbl 0283.53045

[Baird 2009] P. Baird, “A class of three-dimensional Ricci solitons”, Geom. Topol. 13:2 (2009), 979-1015. MR 2010a:53131 Zbl 1157.35465

[Baird and Danielo 2007] P. Baird and L. Danielo, “Three-dimensional Ricci solitons which project to surfaces", J. Reine Angew. Math. 608 (2007), 65-91. MR 2008f:53086 Zbl 1128.53020

[Bakas et al. 2012] I. Bakas, S. Kong, and L. Ni, "Ancient solutions of Ricci flow on spheres and generalized Hopf fibrations", $J$. Reine Angew. Math. 663 (2012), 209-248. MR 2889711 Zbl 1269.53065

[Bando 1987] S. Bando, "Real analyticity of solutions of Hamilton's equation", Math. Z. 195:1 (1987), 93-97. MR 88i:53073 Zbl 0606.58051

[Besse 1988] A. L. Besse, Einstein manifolds, Ergebnisse der Math. und ihrer Grenzgebiete 10, Springer, Berlin, 1988. MR 2008k:53084 Zbl 1147.53001

[Brendle et al. 2011] S. Brendle, G. Huisken, and C. Sinestrari, "Ancient solutions to the Ricci flow with pinched curvature", Duke Math. J. 158:3 (2011), 537-551. MR 2012f:53133 Zbl 1219.53062

[Bryant 2005] R. L. Bryant, "Ricci flow solitons in dimension three with SO(3)-symmetries", preprint, 2005, available at http:// www.math.duke.edu/ bryant/3DRotSymRicciSolitons.pdf.

[Cao 1997] H.-D. Cao, "Limits of solutions to the Kähler-Ricci flow”, J. Differential Geom. 45 (1997), 257-272. MR 99g:53042 Zbl 0889.58067

[Cao and Chen 2012] H.-D. Cao and Q. Chen, "On locally conformally flat gradient steady Ricci solitons", Trans. Amer. Math. Soc. 364:5 (2012), 2377-2391. MR 2888210 Zbl 1245.53038

[Cao et al. 2011] X. Cao, B. Wang, and Z. Zhang, "On locally conformally flat gradient shrinking Ricci solitons", Commun. Contemp. Math. 13:2 (2011), 269-282. MR 2012e:53074 Zbl 1215.53061

[Catino and Mantegazza 2011] G. Catino and C. Mantegazza, "The evolution of the Weyl tensor under the Ricci flow", Ann. Inst. Fourier (Grenoble) 61:4 (2011), 1407-1435. MR 2951497 Zbl 1255.53034

[Catino et al. 2014] G. Catino, C. Mantegazza, and L. Mazzieri, "A note on Codazzi tensors", Math. Ann. (online publication November 2014).

[Chen 2009] B.-L. Chen, "Strong uniqueness of the Ricci flow", J. Differential Geom. 82:2 (2009), 363-382. MR 2010h:53095 Zbl 1177.53036

[Daskalopoulos et al. 2012] P. Daskalopoulos, R. Hamilton, and N. Sesum, "Classification of ancient compact solutions to the Ricci flow on surfaces”, J. Differential Geom. 91:2 (2012), 171-214. MR 2971286 Zbl 1257.53095 
[Eminenti et al. 2008] M. Eminenti, G. La Nave, and C. Mantegazza, "Ricci solitons: the equation point of view", Manuscripta Math. 127:3 (2008), 345-367. MR 2009m:53088 Zbl 1160.53031

[Fateev 1996] V. A. Fateev, "The sigma model (dual) representation for a two-parameter family of integrable quantum field theories", Nuclear Phys. B 473:3 (1996), 509-538. MR 97j:81310 Zbl 0925.81297

[Fateev et al. 1993] V. A. Fateev, E. Onofri, and A. B. Zamolodchikov, "Integrable deformations of the O(3) sigma model: the sausage model”, Nuclear Phys. B 406:3 (1993), 521-565. MR 95d:81111 Zbl 0990.81683

[Feldman et al. 2003] M. Feldman, T. Ilmanen, and D. Knopf, "Rotationally symmetric shrinking and expanding gradient Kähler-Ricci solitons”, J. Differential Geom. 65:2 (2003), 169-209. MR 2005e:53102 Zbl 1069.53036

[Gallot et al. 1990] S. Gallot, D. Hulin, and J. Lafontaine, Riemannian geometry, 2nd ed., Springer, Berlin, 1990. MR 91j:53001 Zbl 0716.53001

[Hamilton 1986] R. S. Hamilton, "Four-manifolds with positive curvature operator", J. Differential Geom. 24:2 (1986), 153-179. MR 87m:53055 Zbl 0628.53042

[King 1990] J. R. King, "Exact similarity solutions to some nonlinear diffusion equations”, J. Phys. A 23:16 (1990), 3681-3697. MR 91j:35062 Zbl 0728.35052

[Kotschwar 2013] B. L. Kotschwar, "A local version of Bando's theorem on the real-analyticity of solutions to the Ricci flow", Bull. Lond. Math. Soc. 45:1 (2013), 153-158. MR 3033963 Zbl 1259.53065

[Lauret 2001] J. Lauret, "Ricci soliton homogeneous nilmanifolds", Math. Ann. 319:4 (2001), 715-733. MR 2002k:53083 Zbl 0987.53019

[Lott 2007] J. Lott, "On the long-time behavior of type-III Ricci flow solutions", Math. Ann. 339:3 (2007), 627-666. MR 2008i:53093 Zbl 1135.53046

[Naber 2010] A. Naber, "Noncompact shrinking four solitons with nonnegative curvature", J. Reine Angew. Math. 645 (2010), 125-153. MR 2012k:53081 Zbl 1196.53041

[Ni 2010] L. Ni, "Closed type I ancient solutions to Ricci flow”, pp. 147-150 in Recent advances in geometric analysis, edited by Y.-I. Lee et al., Adv. Lect. Math. (ALM) 11, International Press, 2010. MR 2011d:53161 Zbl 1198.53074

[Ni and Wallach 2008] L. Ni and N. Wallach, "On a classification of gradient shrinking solitons", Math. Res. Lett. 15:5 (2008), 941-955. MR 2010i:53127 Zbl 1158.53052

[Perelman 2002] G. Perelman, "The entropy formula for the Ricci flow and its geometric applications", preprint, 2002. Zbl 1130.53001 arXiv 0211159

[Perelman 2003] G. Perelman, "Ricci flow with surgery on three-manifolds", preprint, 2003. Zbl 1130.53002 arXiv 0303109

[Petersen and Wylie 2010] P. Petersen and W. Wylie, "On the classification of gradient Ricci solitons", Geom. Topol. 14:4 (2010), 2277-2300. MR 2012a:53060 Zbl 1202.53049

[Rosenau 1995] P. Rosenau, "Fast and superfast diffusion processes", Phys. Rev. Lett. 74 (1995), 1056-1059.

[Zhang 2009a] Z.-H. Zhang, "Gradient shrinking solitons with vanishing Weyl tensor”, Pacific J. Math. 242:1 (2009), 189-200. MR 2010f:53116 Zbl 1171.53332

[Zhang 2009b] Z.-H. Zhang, "On the completeness of gradient Ricci solitons", Proc. Amer. Math. Soc. 137:8 (2009), 2755-2759. MR 2010a:53057 Zbl 1176.53046

Received 8 May 2014. Accepted 9 Feb 2015.

Giovanni CATINO: giovanni.catino@polimi.it

Dipartimento di Matematica, Politecnico di Milano, Piazza Leonardo da Vinci 32, I-20133 Milano, Italy

CARlo MantegazZa: c.mantegazza@sns.it

Scuola Normale Superiore di Pisa, Piazza dei Cavalieri 7, I-56126 Pisa, Italy

LORENZO MAZZIERI: 1.mazzieri@sns.it

Scuola Normale Superiore di Pisa, Piazza dei Cavalieri 7, I-56126 Pisa, Italy 


\title{
Analysis \& PDE
}

\author{
msp.org/apde
}

\section{EDITORS}

EDITOR-IN-CHIEF

\author{
Maciej Zworski \\ zworski@math.berkeley.edu \\ University of California \\ Berkeley, USA
}

BOARD OF EDITORS

Nicolas Burq Université Paris-Sud 11, France

nicolas.burq@math.u-psud.fr

Sun-Yung Alice Chang Princeton University, USA

chang@math.princeton.edu

Michael Christ University of California, Berkeley, USA

mchrist@math.berkeley.edu

Charles Fefferman Princeton University, USA

cf@math.princeton.edu

Ursula Hamenstaedt Universität Bonn, Germany

ursula@math.uni-bonn.de

Vaughan Jones U.C. Berkeley \& Vanderbilt University vaughan.f.jones@vanderbilt.edu

Herbert Koch Universität Bonn, Germany koch@math.uni-bonn.de

Izabella Laba University of British Columbia, Canada ilaba@math.ubc.ca

Gilles Lebeau Université de Nice Sophia Antipolis, France lebeau@unice.fr

László Lempert Purdue University, USA lempert@math.purdue.edu

Richard B. Melrose Massachussets Institute of Technology, USA rbm@math.mit.edu

Frank Merle Université de Cergy-Pontoise, France Frank.Merle@u-cergy.fr

William Minicozzi II Johns Hopkins University, USA minicozz@math.jhu.edu

Werner Müller Universität Bonn, Germany mueller@math.uni-bonn.de
Yuval Peres

Gilles Pisier

Tristan Rivière

Igor Rodnianski

Wilhelm Schlag

Sylvia Serfaty

Yum-Tong Siu

Terence Tao

Michael E. Taylor

Gunther Uhlmann

András Vasy

Dan Virgil Voiculescu

Steven Zelditch
University of California, Berkeley, USA

peres@stat.berkeley.edu

Texas A\&M University, and Paris 6

pisier@math.tamu.edu

ETH, Switzerland

riviere@math.ethz.ch

Princeton University, USA

irod@math.princeton.edu

University of Chicago, USA

schlag@math.uchicago.edu

New York University, USA

serfaty@cims.nyu.edu

Harvard University, USA siu@math.harvard.edu

University of California, Los Angeles, USA tao@math.ucla.edu

Univ. of North Carolina, Chapel Hill, USA met@math.unc.edu

University of Washington, USA gunther@math.washington.edu

Stanford University, USA andras@math.stanford.edu

University of California, Berkeley, USA

dvv@math.berkeley.edu

Northwestern University, USA

zelditch@math.northwestern.edu

PRODUCTION

production@msp.org

Silvio Levy, Scientific Editor

See inside back cover or msp.org/apde for submission instructions.

The subscription price for 2015 is US \$205/year for the electronic version, and \$390/year ( $\$ 55$, if shipping outside the US) for print and electronic. Subscriptions, requests for back issues from the last three years and changes of subscribers address should be sent to MSP.

Analysis \& PDE (ISSN 1948-206X electronic, 2157-5045 printed) at Mathematical Sciences Publishers, 798 Evans Hall \#3840, c/o University of California, Berkeley, CA 94720-3840, is published continuously online. Periodical rate postage paid at Berkeley, CA 94704, and additional mailing offices.

APDE peer review and production are managed by EditFLOw ${ }^{\circledR}$ from MSP.

\section{PUBLISHED BY}

mathematical sciences publishers

nonprofit scientific publishing

http://msp.org/

(C) 2015 Mathematical Sciences Publishers 


\section{ANALYSIS \& PDE}

\section{Volume $8 \quad$ No. $2 \quad 2015$}

Smooth parametric dependence of asymptotics of the semiclassical focusing NLS

SERGEY BELOV and STEPHANOS VENAKIDES

Tunnel effect for semiclassical random walks

JEAN-FrançOIS Bony, FRÉdÉRIC HÉRAU and LAURENT MiCHEL

Traveling wave solutions in a half-space for boundary reactions

Xavier Cabré, Neus Cónsul and José V. Mandé

Locally conformally flat ancient Ricci flows

Giovanni Catino, Carlo MantegazZa and Lorenzo Mazzieri

Motion of thre
regularization

Irene Fonseca, Nicola Fusco, Giovanni Leoni and Massimiliano Morini

Exponential convergence to equilibrium in a coupled gradient flow system modeling

chemotaxis

JoNATHAN ZinSL and DANiEL MATTHES

Scattering for the radial 3D cubic wave equation

BENJAMIN DODSON and ANDREW LAWRIE

Counterexamples to the well posedness of the Cauchy problem for hyperbolic systems 